
\title{
Research S Surate \\ Switchable Gratings for Ultracompact and Ultrahigh Modulation Depth Plasmonic Switches
}

\section{Sandeep Kumar Chamoli}

CIOMP CAS: Changchun Institute of Optics Fine Mechanics and Physics Chinese Academy of Sciences

Mohamed ElKabbash

MIT: Massachusetts Institute of Technology

Chunlei Guo ( $\sim$ guo@optics.rochester.edu )

University of Rochester https://orcid.org/0000-0002-8297-4081

\section{Research Article}

Keywords: Nano-photonics, Plasmonic switch, Phase change materials, Hyperbolic metamaterials

Posted Date: July 29th, 2021

DOI: https://doi.org/10.21203/rs.3.rs-717615/v1

License: (c) (i) This work is licensed under a Creative Commons Attribution 4.0 International License.

Read Full License 


\title{
Switchable gratings for ultracompact and ultrahigh modulation depth plasmonic switches
}

\author{
Sandeep Kumar Chamoli, ${ }^{1,2}$ Mohamed ElKabbash, ${ }^{3 *}$ Chunlei Guo ${ }^{3 *}$ \\ ${ }^{1}$ GPL, State Key Laboratory of Applied Optics, Changchun Institute of Optics, Fine Mechanics and \\ Physics, Chinese Academy of Sciences, Changchun 130033, China \\ ${ }^{2}$ University of Chinese Academy of Science, Beijing 100049, China \\ ${ }^{3}$ The Institute of Optics, University of Rochester, Rochester, New York 14627, USA. \\ *Corresponding authors E-mail: melkabba@ur.rochester.edu; guo@optics.rochester.edu
}

\begin{abstract}
Plasmonic interconnects present a compact platform for high modulation-depth optical switches. Conventional plasmonic switching approaches depend on modifying the dispersion of the surface plasmon polariton (SPP) wave at the metal/dielectric interface. Here, we introduce a novel scheme for ultracompact and high modulation depth (MD) plasmonic switching using a phase-change material-based switchable grating consisting of Antimony Trisulfide $\left(\mathrm{Sb}_{2} \mathrm{~S}_{3}\right)$. In its $\mathrm{ON}$ state, the switchable grating excites surface plasmon polaritons (SPP) and bulk plasmon polaritons (BPPs) in plasmonic films and hyperbolic metamaterials (HMMs), respectively. The SPP switch has a footprint of $23.1 \mu \mathrm{m}^{2}$ and a MD of up to $40 \mathrm{~dB}$. The BPP switch has a footprint of $13.12 \mu \mathrm{m}^{2}$ and a MD of $29.7 \mathrm{~dB}$. The BPP switch enjoys a broadband MD from 299 - $375 \mathrm{THz}$. Concurrently, we show that the same switchable grating on plasmonic film and on HMM is a reflection-based optical switch. Finally, we present a novel scheme for non-local control over the spontaneous emission rate and out-coupled power from emitters embedded in HMMs.
\end{abstract}

Keywords: Nano-photonics, Plasmonic switch, Phase change materials, Hyperbolic metamaterials

\section{Introduction}

Optical switches are central elements in optical interconnect schemes and optical logic gates [1]. The footprint of the stateof-the-art optical switches, based on MEMS technology, is several hundreds of $\mu \mathrm{m}^{2}$ [2]. Plasmonic switches offer a compact alternative to optical switches. The information carriers in plasmonic circuits are surface plasmon polaritons (SPPs); electromagnetic surface waves excited at a metal/dielectric interface. The field associated with SPPs is highly confined and requires a phase matching element, e.g., prisms or diffraction gratings, to couple from free space irradiation. Due to the strong field confinement associated with SPP excitation and their high sensitivity to their surrounding refractive index, highly compact plasmonic switches were demonstrated exploiting the electro-optic [3], magneto-optic effect[4], photo-thermal effect [5], Pauliblocking in graphene [6], phase-change materials[7] and liquid crystals [8]. The aforementioned methods rely on modifying the dispersion of the SPPs in the plasmonic waveguide by either changing the surrounding dielectric properties, e.g., using an electro-optic material and applying a voltage to induce an index change $\Delta \mathrm{n}[2-4,7]$, or through changing the metal's optical properties, e.g., by irradiating it with an ultrafast laser pulse [5]. Therefore, the switching performance becomes intimately dependent on device specific properties such as $\Delta \mathrm{n}$, the field intensity in the modulated region, and the quality factor of the modulated mode.

Here, we propose and numerically investigate a plasmonic switching method that can be applied on various plasmonic platforms using a switchable grating. The proposed switchable grating method can be applied to various plasmonic guided 
mode and plasmonic material. We numerically present a plasmonic switch with a footprint of $23.1 \mu \mathrm{m}^{2}$ and a modulation depth $\left(\mathrm{MD}=10 \log \left(I_{O N} / I_{O F F}\right)\right)$ [9], up to $40 \mathrm{~dB}$. Footprint is simply device area given by the product of total device length with device width. As we have used 10 periods of coupling and outcoupling grating with period of $700 \mathrm{~nm}$ and separated by $1500 \mathrm{~nm}$ so the total device length is $\sim 14.1 \mu \mathrm{m}$ and lateral width taken as $\sqrt{2 \ln (2)} \times$ beam waist size $(1.5 \mu \mathrm{m}) \sim 1.64 \mu \mathrm{m}$, so the SPP switch footprint is $\sim 23.1 \mu \mathrm{m}^{2}$. Moreover, we show that our switch can directly modulate the reflection of an incident beam where photons are information carriers. In addition, we show a novel plasmonic switch based on bulk plasmon polaritons (BPPs) in hyperbolic metamaterials with a footprint of $14 \mu \mathrm{m}^{2}$ as well as a modulation depth of $29.7 \mathrm{~dB}$. For HMM total device length is $\sim 8$ micron and width $\sim 1.64 \mu \mathrm{m}$, so the footprint is $\sim 13.12 \mu \mathrm{m}^{2}$. Finally, we show that our switchable grating can modulate the density of optical states and the collected emission power of quantum emitters embedded inside HMMs.

\section{Results and discussion:}

Figure 1a schematically depicts the proposed plasmonic switch. Our system consists of a coupling grating made of $\mathrm{Sb}_{2} \mathrm{~S}_{3}$, an outcoupling grating and a connecting plasmonic silver $(\mathrm{Ag})$ waveguide. $\mathrm{Ag}$ is chosen due for its low optical losses. Grating parameters such as grating width $\mathrm{w}$, thickness $\mathrm{t}$ and period $\Lambda$ have been optimized to excite SPPs in wavelength ranges where $\mathrm{Sb}_{2} \mathrm{~S}_{3}$ has low optical losses. Perfectly matched layer (PML) is chosen as a boundary condition in our $2-\mathrm{D}$ simulation for all the boundaries. A TM polarised gaussian light source with $2 \mu m$ waist size from $1000 \mathrm{~nm}-1200 \mathrm{~nm}$ is used to illuminate the coupling grating for excitation purpose. A metallic substrate can support SPP at the metal-dielectric interface if the phasematching condition is satisfied. A coupling grating with period $\Lambda$ can couple the incident radiation at an angle $\theta$ to SPPs if: $k_{s p p}=k_{0} n_{e n v} \sin \theta+m \frac{2 \pi}{\Lambda}$, where $k_{s p p}$ is SPP wavevector, $k_{0}=\frac{2 \pi}{\lambda_{0}}$ is the wave vector in free space, $n_{e n v}$ is refractive index of the incidence medium. In our case, the grating consists of a phase change material $\left(\mathrm{Sb}_{2} \mathrm{~S}_{3}\right)$ embedded in a dielectric with refractive index $n_{e n v}$. $\mathrm{Sb}_{2} \mathrm{~S}_{3}$ can switch its phase from crystalline to amorphous optically, using an ultrafast pulsed laser, [9] or thermally, e.g., using ITO or graphene microheaters below the phase change material [10, 11]. The switching rate from the amorphous to crystalline phases of $\mathrm{Sb}_{2} \mathrm{~S}_{3}$ is $\sim 10 \mathrm{MHz}$ which is relatively fast compared to existing optical switches $[12,13]$. The grating is designed such that the refractive index of $\mathrm{Sb}_{2} \mathrm{~S}_{3}$ in the amorphous phase is approximately equal to $n_{e n v}$. When $\mathrm{Sb}_{2} \mathrm{~S}_{3}$ is in the crystalline phase, the grating is capable of exciting SPPs which travels along the metal-dielectric interface and eventually radiates through the out-coupling grating. When electromagnetic energy is transferred to SPPs, the reflected light from the coupling grating experiences a sudden decrease (reflection anomaly). On the other hand, when $\mathrm{Sb}_{2} \mathrm{~S}_{3}$ is in the amorphous phase, the index contrast with the environment is negligible and the coupling grating effectively disappears. We thus have a plasmonic switch and an optical switch. The plasmonic switch is in the ON state, where light detected from the outcoupling grating when the coupling grating is activated, i.e., when $\mathrm{Sb}_{2} \mathrm{~S}_{3}$ is in the crystalline phase. The optical switch is in the ON state when the coupling grating is not activated, i.e., when $\mathrm{Sb}_{2} \mathrm{~S}_{3}$ is in the amorphous phase, and light is reflected back from the coupling grating as it cannot excite SPPs. We numerically investigate our proposed approach using a finite difference time domain (FDTD) commercial software Lumerical ${ }^{\circ}$. The coupling grating is made of $\mathrm{Sb}_{2} \mathrm{~S}_{3}$ [13] embedded in a dielectric environment with a refractive index $n_{e n v}=2.8$. Possible choices for the dielectric environment are rutile $\mathrm{TiO}_{2}(\mathrm{n} \sim 2.8)$ and $\mathrm{SiC}(\mathrm{n} \sim 2.6)$. The $1 \mathrm{D}$ grating period, width and thickness are $800 \mathrm{~nm}, 100 \mathrm{~nm}$, and $500 \mathrm{~nm}$, respectively. The metallic substrate is $\mathrm{Ag}$ (permittivity values obtained from chemical rubber company (CRC) press) [14]. The outcoupling grating is made of $\mathrm{SiO}_{2}$ and placed 1.5 micron away from the coupling grating with a period, width and thickness of $700 \mathrm{~nm}, 100 \mathrm{~nm}$, and $300 \mathrm{~nm}$, respectively. Normal incident Gaussian source illumination and perfectly matched boundaries were used. Two power monitors were used to detect reflected light from the coupling grating and outcoupled light from the outcoupling grating.

Figure $1 \mathrm{~b}$ shows the transverse electric field $\mathrm{E}_{\mathrm{x}}$ component distribution for $\mathrm{Sb}_{2} \mathrm{~S}_{3}$ in the crystalline phase at resonacne wavelength of $1083 \mathrm{~nm}$. The grating successfully excited an SPP with its characteristic exponential decay in the propagation direction and localization at the metal/dielectric interface. On the other hand, when $\mathrm{Sb}_{2} \mathrm{~S}_{3}$ is switched to its amorphous phase, the grating is not activated, i.e., effectively disappears, and no SPP is excited as shown in Fig. 1c. The calculated SPP modulation depth reaches $40 \mathrm{~dB}$ at $\lambda_{0}=1138 \mathrm{~nm}$ (see figure $1(\mathrm{~d})$ ). The modulation depth is given by $10 \log \left(I_{O N} / I_{O F F}\right)$, where $I_{O N}$ and $I_{O F F}$ are the signals detected using the outcoupling grating in case crystalline and amorphous $\mathrm{Sb}_{2} \mathrm{~S}_{3}$ coupling grating, respectively.

In addition to realizing an SPP switch, a direct consequence of our approach is realizing a reflection optical switch. For light incident on the coupling grating, when the grating is switched ON, excitation of SPPs is associated with a dip in reflection within the wavelength range where the grating phase matching condition is satisfied. By monitoring the reflection from the coupling grating, we observe $\Delta \mathrm{R} \sim 0.57$ is at $\lambda_{0} \sim 1083 \mathrm{~nm}$ (see figure $1 \mathrm{e}$ ). 

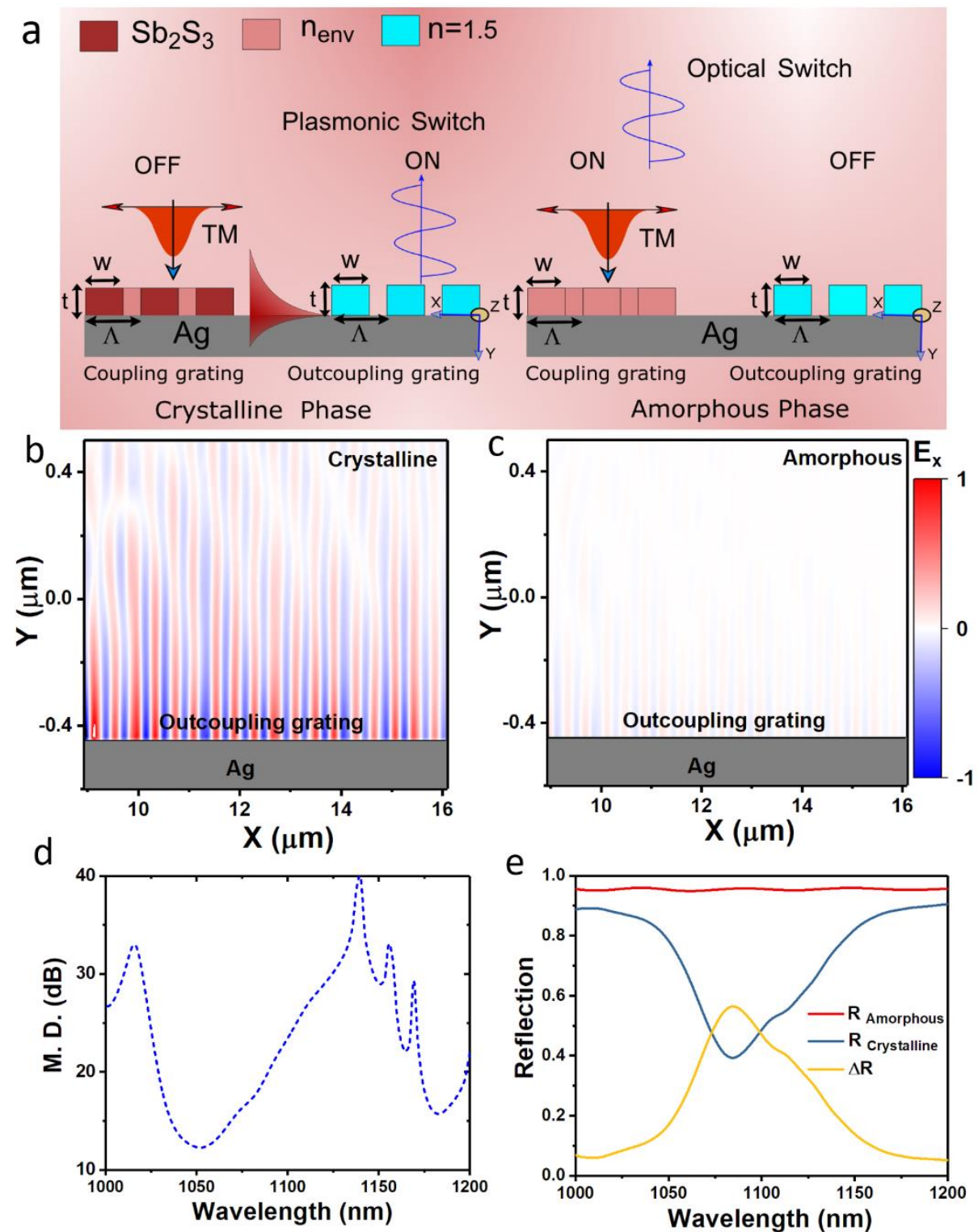

Figure 1: (a) Schematics of the proposed switchable grating as an SPP switch. When $\mathrm{Sb}_{2} \mathrm{~S}_{3}$ is in its crystalline phase, a substantial index contrast with the dielectric environment exists and the grating is activated. When $\mathrm{Sb}_{2} \mathrm{~S}_{3}$ is in its amorphous state, however, the index contrast reduces significantly, and the grating disappears. The design introduces a optical switch operating based on the reflected light from the coupling grating and a plasmonic switch operating based on light collected from the outcoupling grating. Note that when the photonic switch is in the ON state, the plasmonic switch is in the OFF state and vice versa. FDTD calculation of the electric field $\mathrm{E}_{\mathrm{x}}$ component for the switchable grating at $1083 \mathrm{~nm}$ captured in the region after coupling grating in the (b) Crystalline state and (c) Amorphous state. SPP is only excited and then outcoupled to radiation when the grating is in its crystalline state. (d) Modulation depth of the plasmonic switch as a function of wavelength based on light collected from the outcoupling grating. We Calculated reflection and reflection difference collected from the coupling grating for photonics switch. Here, $\Delta \mathrm{R}$ is reflection difference between the crystalline and amorphous phase, $\Delta \mathrm{R}=\mathrm{R}_{\mathrm{Amorphous}}$

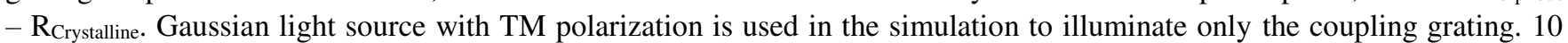
coupling and outcoupling grating periods have been used in the simulation.

We adopt a similar approach to optically switch the high-k modes of hyperbolic metamaterials (HMMs), i.e., bulk plasmon polaritons (BPP)[15]. HMMs are artificially engineered material with a hyperbolic iso-frequency curve. A common platform 
for HMMs is metal-dielectric thin film stack where the uniaxial anisotropic permittivity values are such that $\varepsilon_{\|}=\varepsilon_{\mathrm{x}}=\varepsilon_{\mathrm{y}}<0$ and $\varepsilon_{\perp}=\varepsilon_{\mathrm{Z}}>0$. The positive perpendicular permittivity is due to the ability of these HMMs to excite bulk modes that consist of evanescently coupled SPPs, i.e., BPPs.

Because the local density of optical states (LDOS) are related to the volume enclosed by the iso-frequency surface[16], HMMs enjoy high LDOS which leads to various effects enhanced Purcell factors or quantum emitters[16], perfect light absorption [17, 18], and long-range dipole-dipole interactions [19]. HMM-based optical switches relied on switching the topological transition frequency using an HMM with an active element, e.g., a phase change material or graphene [20, 21]. In these works, however, BPP was not the information carrier, rather the reflected photons from the HMM. By using a switchable coupling grating and placing an out-coupling grating at the bottom of the HMM [22], we can create an optical switch based on BPPs (Fig. 2). The coupling grating consists of $\mathrm{Sb}_{2} \mathrm{~S}_{3}$ embedded in a dielectric environment with a refractive index $n_{\text {env }}=2.8$. The grating parameters such as grating width $w$, grating thickness $t$ and grating period $\Lambda$ are optimized to have resonance in the region where $\mathrm{Sb}_{2} \mathrm{~S}_{3}$ losses are negligble. The optimized grating period, width and thickness are $1000 \mathrm{~nm}, 500 \mathrm{~nm}$ and 310 $\mathrm{nm}$. The outcoupling grating refractive index is 1.5 and has the same dimensions as the coupling grating.
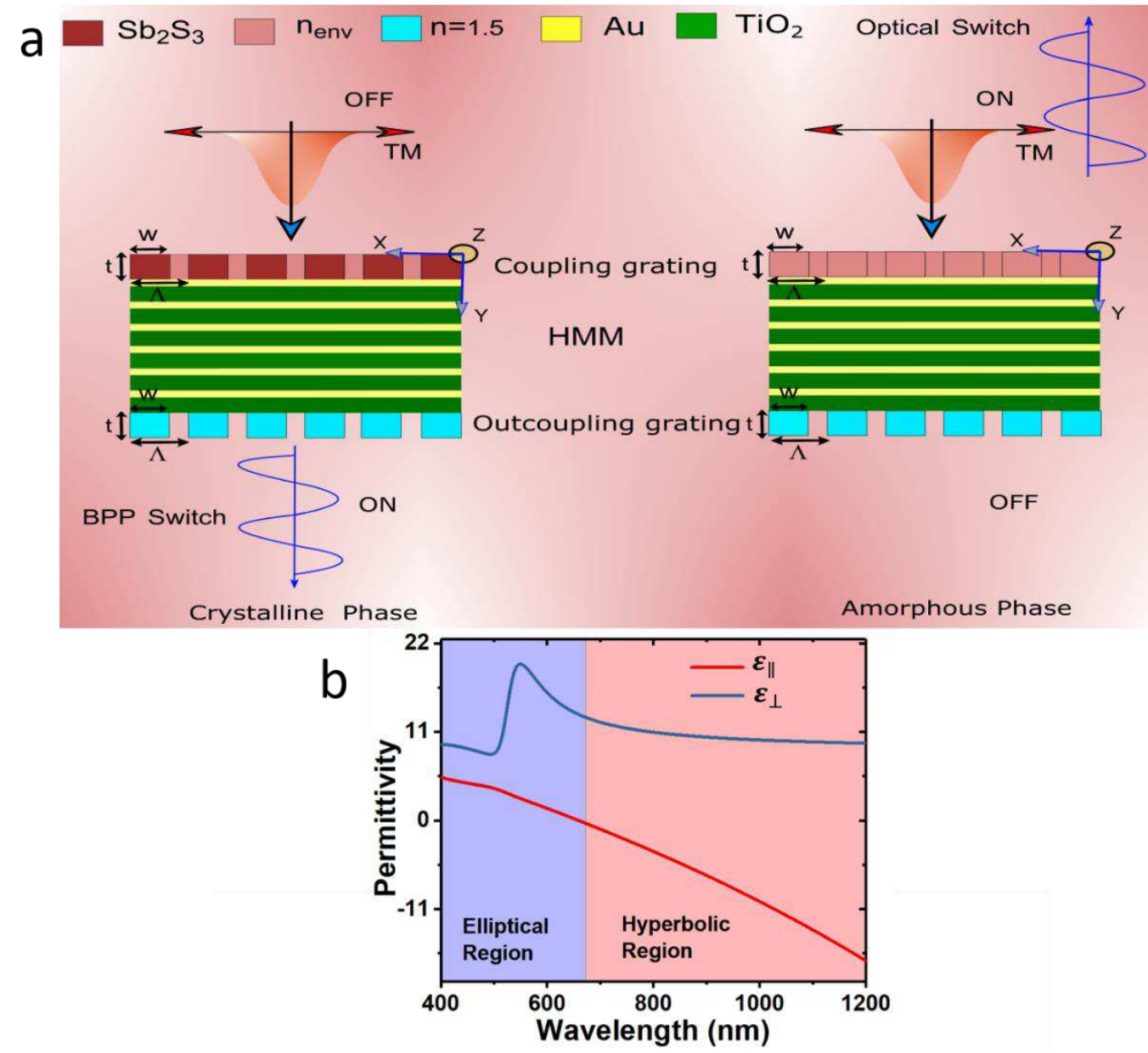

Figure 2: (a) Schematics of the BPP and optical switch consisting of a switchable grating on an HMM and an outcoupling grating at bottom. The HMM consists of a stack of $\mathrm{Au}$ (yellow) and $\mathrm{TiO}_{2}$ (green) bilayers. When $\mathrm{Sb}_{2} \mathrm{~S}_{3}$ is in its crystalline phase, a substantial index contrast with the dielectric environment exists and the grating is activated. When $\mathrm{Sb}_{2} \mathrm{~S}_{3}$ is in its amorphous state, however, the index contrast reduces significantly, and the grating disappears. The design introduces a optical switch operating based on the reflected light from the coupling grating and a BPP switch operating based on light collected from the outcoupling grating. Note that when the optical switch is in the ON state, the BPP switch is in the OFF state and vice versa. Gaussian light source with TM polarization focused on coupling grating on the top to excite the BPP. 10 coupling and outcoupling grating periods have been used in the simulation. (b) Effective parallel $\left(\varepsilon_{\|}\right)$and perpendicular $\left(\varepsilon_{\perp}\right)$ permittivity of $\mathrm{HMM}$ for $5 \mathrm{~nm} \mathrm{Au}$ and $10 \mathrm{~nm} \mathrm{TiO}_{2}$ unit cell. 
Our HMM consists of 24 bilayers of $\mathrm{Au}(5 \mathrm{~nm})$ and $\mathrm{TiO}_{2}(10 \mathrm{~nm})$ thin films. The perpendicular and parallel permittivity of the system are given by $\varepsilon_{\|}=f_{m} \varepsilon_{m}+f_{d} \varepsilon_{d}$ and $\varepsilon_{\perp}=\frac{\varepsilon_{m} \varepsilon_{d}}{f_{m} \varepsilon_{m}+f_{d} \varepsilon_{d}}$ respectively and calculated in figure 2 (b) [15]. The topological transition occurs when $\varepsilon_{\perp}<0$ at $\sim 600 \mathrm{~nm}$ as shown in Fig. 2 (b). Figure 3 a show the calculated electric field intensity for the HMM. When the grating is in the crystalline phase, BPPs are excited inside the HMM. Conversely, when the grating is in the amorphous phase, BPPs are not excited (see Fig. 3 b). Nearly $29.7 \mathrm{~dB}$ modulation depth of BPPs at $\sim 900 \mathrm{~nm}$ (see figure 3 (c)) is obtained. Moreover, high MD is obtained over a wide range of wavelengths $(800 \mathrm{~nm}-1000 \mathrm{~nm})$ with an average MD of $18 \mathrm{~dB}$. However, the ultrahigh MD comes at the cost of increasing the insertion loss. The insertion loss of the BPP switch is $-33 \mathrm{~dB}$ while for SPP switch is less than $10 \mathrm{~dB}$. Moreover, due to the high LDOS of HMMs, the reflection drop associated with switching the grating $\mathrm{ON}$ and exciting BPPs is stronger than the case of SPPs. Consequently, we obtain a $\Delta \mathrm{R}$ of $\sim 0.55$ at $750 \mathrm{~nm}$ as shown in Fig. $3 \mathrm{~d}$. The calculated effective index of fundamental mode at $750 \mathrm{~nm}$ is $2.7+i 0.012 \mathrm{using}$ FDE solver in Lumerical mode solution. The effective index calculation suggests the grating period in order to excite the BPP mode [23].
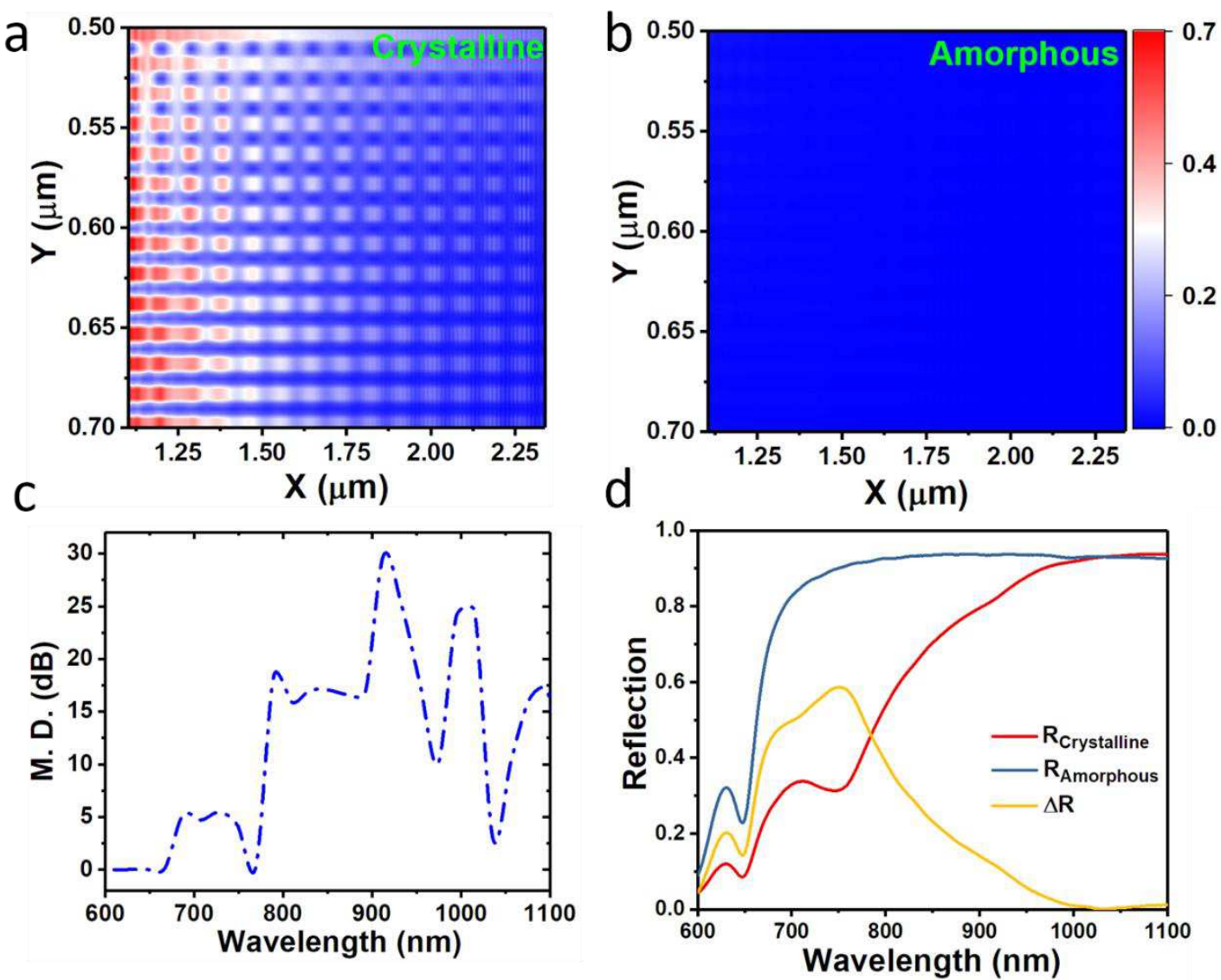

Figure 3: Simulated field intensity in the HMM when the coupling switchable grating is in its (a) Crystalline (ON) state and (b) Amorphous (OFF) state. (c) Modulation depth of the BPP plasmonic switch as a function of wavelength based on light collected from the outcoupling grating below. (d) Calculated reflection and reflection difference collected from the coupling grating on top for photonics switch, where $\Delta \mathrm{R}=\mathrm{R}_{\text {Amorphous }}-\mathrm{R}_{\text {crystalline. }}$

The high LDOS of HMMs can accelerate the spontaneous emission rate of quantum emitters coupled via their near fields to the HMMs high-k modes [15]. Emitters located inside the HMM excite BPPs through their near field. Therefore, the emission remains mostly trapped and eventually dissipates because of the momentum mismatch between BPPs and free space radiation. Using a grating is essential to out-couple the emission of emitters embedded inside HMMs [24]. We show here that our switchable grating can be used to control the coupling of quantum emitters to radiation and to nonlocally control the emitter's spontaneous emission rate. Figure 4a schematically shows our calculation setup where a dipole is placed within the HMM. When the grating is switched ON in crystalline phase, the emission out-couples from the HMM, i.e., the system behaves as a switch for emission collected from quantum emitters as shown in electric field profile of the emitted radiation (Fig. 4 (b)). 
However, in the amorphous phase, where the grating effectively disappears, the emission trapped inside the HMM (Fig. 4 (c)). Figure $4 \mathrm{~d}$ shows a high modulation depth of up to $31 \mathrm{~dB}$ when grating is in crystalline phase i.e., ON state. We note that the HMM increases the Purcell factor of the emitter due to the HMM high local density of optical states (LDOS). Because the LDOS depends on the number of propagating and evanescent states available to the emitter to radiate into, when the grating is $\mathrm{ON}$, the Purcell factor significantly increases by $100 \%$ (from 150 to 300 at $840 \mathrm{~nm}$ ). Moreover, only when the grating is ON, i.e., $\mathrm{Sb}_{2} \mathrm{~S}_{3}$ in the crystalline phase, the emission is out coupled and detected outside the HMM as shown in Fig. $4 \mathrm{e}$.

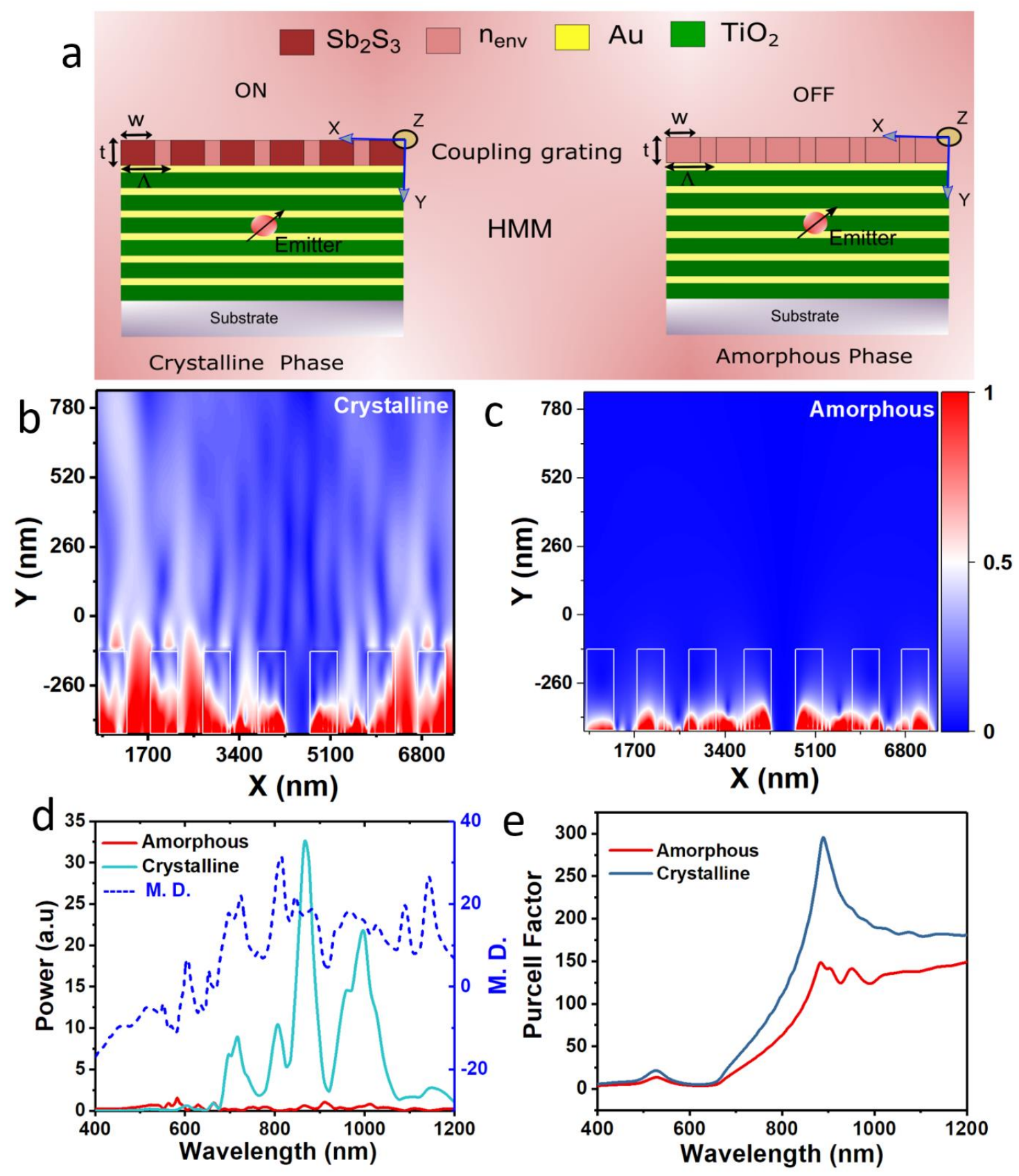

Figure 4: (a) Schematics of an emitter (vertical dipole) embedded in an HMM with a switchable grating to non-locally control the spontaneous emission rate of the emitter. When $\mathrm{Sb}_{2} \mathrm{~S}_{3}$ is in its crystalline phase, a substantial index contrast with the dielectric environment exists and the grating is activated. When $\mathrm{Sb}_{2} \mathrm{~S}_{3}$ is in its amorphous state, however, the index contrast reduces significantly, and the grating disappears. The thickness of $\mathrm{Au}$ and $\mathrm{TiO}_{2}$ are $5 \mathrm{~nm}$ and $10 \mathrm{~nm}$. The grating parameters are : $\mathrm{w}=500 \mathrm{~nm}, \mathrm{t}=310 \mathrm{~nm}$ and $1000 \mathrm{~nm}, \mathrm{n}_{\text {env }}=2.8$. Electric field profile in (b) crystalline phase and (c) amorphous phase, coupling grating is shown with white rectangles. (d) Calculated far field power radiated in the crystalline (ON) and amorphous (OFF) phase and modulation depth. (e) The calculated Purcell factor in the crystalline and amorphous phase. 


\section{Conclusion:}

In conclusion, we proposed a method for a plasmonic switch that controls the excitation of SPPs in plasmonic films and BPPs in HMMs. The SPP switch enjoys a footprint of $23.1 \mu^{2}, 40 \mathrm{~dB}$ MD and less than $10 \mathrm{~dB}$ insertion loss, while the BPP switch has a footprint of $13.1 \mu \mathrm{m}^{2}, 29.7 \mathrm{~dB}$ MD, and $-33 \mathrm{~dB}$ insertion loss. The small footprint of the proposed plasmonic switches makes them as building blocks of high space-bandwidth spatial light modulators[25] Finally, we showed that our approach can non-locally control the LDOS and outcoupling of spontaneous emission from quantum emitters coupled to HMMs high-k modes with an MD reaching $31 \mathrm{~dB}$. The plasmonic switch offers an attractive option for ultracompact and high modulation depth applications. The proposed concept can be extended to tunable metasurfaces[26], e.g., for tunable beam deflection, guided mode resonances and bound state in the continuum modes [27] .

we finally note that optical losses are the biggest problem facing all plasmonic devices and switches. Unless new plasmonic materials appear that exhibit low optical losses, it will remain a niche alternative where significantly decreasing the device footprint is desirable as shown in our work. For instance, an important metric for spatial light modulators, which can consist of an array of optical switches, is the space-bandwidth product which is represented by the product of the pixel footprint of individual optical switches in an SLM and the largest spatial size over which these pixels can extend. The space- bandwidth product corresponds to the total number of parallel channels of data that the SLM can encode and hence is an important performance metric [25]. Operating at longer wavelengths using doped semiconductors[28], adding gain material [29, 30], discovering of low loss plasmonic materials [27] or exciting other types of lower loss plasmonic modes such as long-range surface plasmons [31], are all possible ways to reduce losses.

\section{Declarations}

Ethical Statement Hereby, I Sandeep Chamoli, consciously assures that for the manuscript titled "Switchable gratings for ultracompact and ultrahigh modulation depth plasmonic switches," the following is fulfilled:

1. This material is the authors' own original work, which has not been previously published elsewhere.

2. The paper is not currently being considered for publication elsewhere.

3. The paper reflects the authors' own research and analysis in a truthful and complete manner.

4. The paper properly credits the meaningful contributions of co-authors and co-researchers.

5. The results are appropriately placed in the context of prior and exist-ing research.

6. All sources used are properly disclosed (correct citation). Literally copying of text must be indicated as such by using quotation marks and giving proper reference.

7. All authors have been personally and actively involved in substantial work leading to the paper, and will take public responsibility for its content.

The violation of the Ethical Statement rules may result in severe con-sequences.

I agree with the above statements and declare that this submission follows the policies of Plasmonics Journal as outlined in the Guide for Authors and in the Ethical Statement.

Author Contribution: Conceptualization, M. E. and S. K. C.; methodology, M. E. and S. K. C.; software, S. K. C. and M. E.; validation, S.K.C., M.E. and C.G.; formal analysis, S. K. C.; investigation, S. K. C. and M. E.; writing original draft preparation, M. E. and S. K. C.; writing review and editing, S.K.C., M.E. and C. G.; supervision, C. G.; project administration, C. G.;

Consent to Participate Informed consent was obtained from all individual participants included in the study.

Consent for PublicationThere are no case studies involve in the current work. Therefore, this declaration is not applicable in our case.

Conflict of Interest The authors declare no competing interests

Funding: National Natural Science Foundation (11674178); Jilin Provincial Science and Technology Development Project (20180414019GH); Bill \& Melinda Gates Foundation (INV-009181).

Acknowledgment: S. K. C. acknowledges his sponsorship by "CAS-TWAS Presidential Fellowship for international doctorate students". 
Availability of data: The data that support the findings of this study are available from the corresponding author upon reasonable request. The data for this research work is available upon request.

Code Availability: The code that support the findings of this study are available upon reasonable request.

\section{References}

[1] X. Zhang, J. Yang, Ultrafast plasmonic optical switching structures and devices, Frontiers in Physics, 7 (2019) 190.

[2] A. Emboras, C. Hoessbacher, C. Haffner, W. Heni, U. Koch, P. Ma, Y. Fedoryshyn, J. Niegemann, C. Hafner, J. Leuthold, Electrically controlled plasmonic switches and modulators, IEEE Journal of Selected Topics in Quantum Electronics, 21 (2014) 276-283.

[3] W. Cai, J.S. White, M.L. Brongersma, Compact, high-speed and power-efficient electrooptic plasmonic modulators, Nano letters, 9 (2009) 4403-4411.

[4] A. Haddadpour, V.F. Nezhad, Z. Yu, G. Veronis, Highly compact magneto-optical switches for metal-dielectric-metal plasmonic waveguides, Opt. Lett., 41 (2016) 4340-4343.

[5] K.F. MacDonald, Z.L. Sámson, M.I. Stockman, N.I. Zheludev, Ultrafast active plasmonics, Nature Photonics, 3 (2009) 55-58.

[6] M. Ono, M. Hata, M. Tsunekawa, K. Nozaki, H. Sumikura, H. Chiba, M. Notomi, Ultrafast and energy-efficient alloptical switching with graphene-loaded deep-subwavelength plasmonic waveguides, Nature Photonics, 14 (2020) $37-43$. [7] S.-Y. Lee, Design of a plasmonic switch using ultrathin Chalcogenide phase-change material, Current Optics and Photonics, 1 (2017) 239-246.

[8] Yan Jun Liu, Yue Bing Zheng, Jinjie Shi, Hua Huang, Thomas R. Walker, and Tony Jun Huang, "Optically switchable gratings based on azo-dye-doped, polymer-dispersed liquid crystals," Opt. Lett. 34, 2351-2353 (2009)

[9] G.T. Reed, G. Mashanovich, F.Y. Gardes, D. Thomson, Silicon optical modulators, Nature photonics, 4 (2010) $518-526$.

[10] C. Ríos, Y. Zhang, S. Deckoff-Jones, H. Li, J. B. Chou, H. Wang, M. Shalaginov, C. Roberts, C. Gonçalves, V. Liberman, T. Gu, J. Kong, K. Richardson, and J. Hu, "Reversible switching of optical phase change materials using graphene microheaters," Opt. InfoBase Conf. Pap. Part F129-, 10-11 (2019).

[11] Y.-Y. Au, H. Bhaskaran, and C. D. Wright, "Phase-change devices for simultaneous optical-electrical applications," Sci. Rep. 7(1), (2017), 9688.

[12] S. K. Chamoli, G. Verma, S. Singh, C. Guo, Phase change material-based nano-cavity as an efficient optical modulator, Nanotechnology, 32, (2021), 095207.

[13] M. Delaney, I. Zeimpekis, D. Lawson, D.W. Hewak, O.L. Muskens, A New Family of Ultralow Loss Reversible Phase Change Materials for Photonic Integrated Circuits: $\mathrm{Sb}_{2} \mathrm{~S}_{3}$ and $\mathrm{Sb}_{2} \mathrm{Se}_{3}$, Advanced Functional Materials, DOI (2020) 2002447.

[14] CRC Handbook of Chemistry and Physics. Cleveland, Ohio: CRC Press, 1977.

[15] S.K. Chamoli, M. ElKabbash, J. Zhang, C. Guo, Dynamic control of spontaneous emission rate using tunable hyperbolic metamaterials, Opt. Lett., 45 (2020) 1671-1674.

[16] H.N. Krishnamoorthy, Z. Jacob, E. Narimanov, I. Kretzschmar, V.M. Menon, Topological transitions in metamaterials, Science, 336 (2012) 205-209.

[17] C. Guclu, S. Campione, F. Capolino, Hyperbolic metamaterial as super absorber for scattered fields generated at its surface, Physical Review B, 86 (2012) 205130.

[18] E.E. Narimanov, H. Li, Y.A. Barnakov, T. Tumkur, M. Noginov, Reduced reflection from roughened hyperbolic metamaterial, Opt. Express, 21 (2013) 14956-14961.

[19] W.D. Newman, C.L. Cortes, A. Afshar, K. Cadien, A. Meldrum, R. Fedosejevs, Z. Jacob, Observation of long-range dipole-dipole interactions in hyperbolic metamaterials, Science advances, 4 (2018) eaar5278.

[20] W. Liang, Z. Li, Y. Wang, W. Chen, Z. Li, All-angle optical switch based on the zero-reflection effect of graphenedielectric hyperbolic metamaterials, Photonics Research, 7 (2019) 318-324.

[21] M. Shoaei, M.K. Moravvej-Farshi, L. Yousefi, All-optical switching of nonlinear hyperbolic metamaterials in visible and near-infrared regions, JOSA B, 32 (2015) 2358-2365.

[22] T. Xu, H.J. Lezec, Visible-frequency asymmetric transmission devices incorporating a hyperbolic metamaterial, Nature Communications, 5 (2014) 4141.

[23] Sreekanth, K., De Luca, A. \& Strangi, G. Experimental demonstration of surface and bulk plasmon polaritons in hypergratings. Sci Rep 3, 3291 (2013).

[24] Lu, D., Kan, J., Fullerton, E. et al. Enhancing spontaneous emission rates of molecules using nanopatterned multilayer hyperbolic metamaterials. Nature Nanotech 9, 48-53 (2014). 
[25] J.A. Neff, R.A. Athale, S.H. Lee, Two-dimensional spatial light modulators: a tutorial, Proceedings of the IEEE, 78 (1990) 826-855.

[26] C. Jin, J. Zhang, C. Guo, Metasurface integrated with double-helix point spread function and metalens for threedimensional imaging, Nanophotonics, 8 (2019) 451-458.

[27] Hsu, C., Zhen, B., Stone, A. et al. Bound states in the continuum. Nat Rev Mater 1, 16048 (2016).

[28] J.B. Khurgin, How to deal with the loss in plasmonics and metamaterials, Nature Nanotechnology, 10 (2015) 2-6.

[29] M. El Kabbash, A. Rahimi Rashed, K.V. Sreekanth, A. De Luca, M. Infusino, G. Strangi, Plasmon-exciton resonant energy transfer: across scales hybrid systems, Journal of Nanomaterials, 2016 (2016).

[30] M. ElKabbash, A.R. Rashed, B. Kucukoz, Q. Nguyen, A. Karatay, G. Yaglioglu, E. Ozbay, H. Caglayan, G. Strangi, Ultrafast transient optical loss dynamics in exciton-plasmon nano-assemblies, Nanoscale, 9 (2017) 6558-6566.

[31] P. Berini, Long-range surface plasmon polaritons, Advances in optics and photonics, 1 (2009) 484-588. 